\title{
Steps Toward Identifying Central Bank Policy Preferences*
}

\author{
Richard Dennis
}

May 2000

\section{Federal Reserve Bank of San Francisco}

\section{JEL: C15, E52.}

Keywords: Policy Regimes, Identification.

\begin{abstract}
This paper takes the parameters in central bank loss functions as fundamental preferences to be estimated from the data. It is these preferences (along with target values) that define the policy regime in operation and that potentially change with senior central bank appointments. Optimizing central banks apply policy rules whose feedback coefficients are functions of its preferences. Consequently, under some conditions, it is possible to back out estimates of the preference parameters from estimated policy reaction functions. This paper establishes conditions under which a policy regime can be identified and illustrates these conditions using a number of popular models.
\end{abstract}

\footnotetext{
* I am grateful to Vlad Pavlov, Graeme Wells, and especially Adrian Pagan for comments.

- Address for correspondence: Economic Research Department, Federal Reserve Bank of San Francisco, 101 Market St, San Francisco, CA 94105, USA. Email: Richard.Dennis@sf.frb.org. The views expressed in this paper do not necessarily reflect those of the Federal Reserve Bank of San Francisco or the Federal Reserve System.
} 


\section{1) Introduction}

Modern analyses of central bank behavior begin with a policy objective function and construct policy rules by optimizing the objective function subject to a system of constraints. Descriptions of actual central bank behavior can also be obtained by estimating policy reaction functions directly. For the United States, Clarida, Gali, and Gertler (1998), Fuhrer (1997), and Judd and Rudebusch (1998) have all estimated reaction functions for the Federal Reserve. Taylor (1993) also developed a rule describing Federal Reserve policy decisions, popularly known as the Taylor rule. Clearly these estimated policy reaction functions and those developed through optimization are not unrelated. Optimal policy rules set the policy instrument as a linear function of the state vector. The feedback coefficients in these optimal rules are nonlinear functions of the parameters in the model constraining the optimization, as well as the parameters in the policy objective function. In principle it is these nonlinear parameter combinations that applied studies estimate.

A better understanding of monetary policy decisions can be had if the monetary authority's preferences can be disentangled and extracted from estimated policy rules. With these preferences in hand we would know which variables enter the policy objective function; which aspects of the economy the central bank is concerned about; and how senior central bank appointments affect the policy regime in operation. Because they relate directly to the policy regime in place, policy preferences, not estimated policy rules, are more informative of the objectives and incentives underpinning policy decisions.

Given a plausible economic model, and provided the estimated policy rule is the outcome of a constrained optimization process, it should be possible to find objective function parameters such that the optimal rule closely resembles the estimated rule. Of course, if these implied policy objective function parameters are to be informative it is important that the model constraining central bank behavior realistically capture the relationships at 
work in the economy. The objective of this paper is to present conditions under which a policy regime in operation can be uncovered from the data. ${ }^{1}$

One of the most common objective functions employed in the monetary policy rules literature defines loss in terms of a linear combination of the unconditional variances of a vector of economic variables. ${ }^{2}$ To formalize this, let $\mathbf{z}_{\mathbf{t}}$ be a vector of economic variables, including the policy instrument(s). ${ }^{3}$ We assume that $\mathbf{z}_{\mathbf{t}}$ is weakly stationary with unconditional mean vector $\mathbf{z}_{\mathbf{t}}^{*}$. Each element in $\mathbf{z}_{\mathbf{t}}$ has its counterpart in $\mathbf{z}_{\mathbf{t}}^{*}$. Without loss of generality $\mathbf{z}_{\mathbf{t}}{ }_{\mathbf{t}}$ is taken to equal the null vector. ${ }^{4}$ Further, it is assumed that policy makers target the unconditional mean of $\mathbf{z}_{\mathbf{t}}$, and therefore that $\mathbf{z}_{\mathbf{t}}^{*}$ is also the target vector. With this notation every variable in $\mathbf{z}_{\mathbf{t}}$ has a nominal target value. Of course, for many of these variables zero weight may be applied to their deviations from target in the objective function.

Denote the unconditional variance-covariance matrix of $\mathbf{z}_{\mathbf{t}}$ by $\Omega$. Let $\mathbf{W}$ be a symmetric, positive semi-definite, matrix of policy weights; $\Omega$ and $\mathbf{W}$ share the same dimensions. The infinite horizon policy objective function is: $\operatorname{Loss}[0, \infty]=\operatorname{tr}[\mathbf{W} \Omega]$, where ' $\operatorname{tr}$ ' is the trace operator. In many applications $\mathbf{W}$ is a diagonal matrix. Given this objective function, a policy regime is defined by the matrix of policy weights (preferences), $\mathbf{W}$, and the vector of targets, $\mathbf{z}_{\mathbf{t}}^{*}$. It is the elements in this $\mathbf{W}$ matrix that we seek to identify.

The structure of the paper is as follows. Section 2 develops the general economic structure within which subsequent analysis takes place. Using this general economic

${ }^{1}$ Soderlind (1999) estimates the parameters in an objective function using a model of the United States. He does not consider identification however.

2 See, for example, Ball (1999), Svensson (1999), Svensson (1998), Fair and Howrey (1996), Rudebusch and Svensson (1998), Clarida, Gali, and Gertler (1999), Fuhrer (1997), and Bharucha and Kent (1998), among others.

3 Throughout this paper we use bolded capitals for matrices, bolded lowercase for vectors, and lowercase for scalars.

${ }^{4}$ Normalizing $\mathbf{z}_{\mathbf{t}}^{*}$ to equal zero is without loss of generality when policy decisions are constrained by a system of linear equality constraints. This normalization is not appropriate if some of the constraints are inequality constraints, such as a constraint preventing the nominal interest rate from going negative. 
framework section 3 systematically examines the conditions under which policy preferences can be identified. To illustrate how the identification conditions are applied in practice section 4 considers several popular models and examines whether their structure permits identification of the policy regime. Section 5 concludes.

\section{2) A General Macroeconomic Setting}

Consider the following macroeconomic specification:

$$
\mathbf{A}_{0} \mathbf{y}_{\mathbf{t}}=\mathbf{A}_{1} \mathbf{y}_{\mathrm{t}-1}+\mathbf{A}_{2} \mathrm{E}_{\mathrm{t}-1} \mathbf{y}_{\mathrm{t}+\mathbf{1}}+\mathbf{A}_{3} \mathbf{x}_{\mathrm{t}}+\mathbf{v}_{\mathrm{t}}, \quad \mathbf{v}_{\mathrm{t}} \sim \operatorname{iid}[\mathbf{0}, \Sigma]
$$

where $\mathbf{y}_{\mathbf{t}}$ is an $\mathrm{n} \times 1$ vector of economic variables, $\mathbf{x}_{\mathbf{t}}$ a $\mathrm{p} \times 1$ vector of policy instruments, $\mathbf{v}_{\mathbf{t}}$ an $n \times 1$ vector of stochastic innovations, and $E_{t-1}$ is the mathematical expectations operator conditional upon information set $I_{t-1}$, where $I_{t}=\left\{\mathbf{y}_{t}, \mathbf{x}_{t}, I_{t-1}\right\}$. Matrices $\mathbf{A}_{\mathbf{0}}, \mathbf{A}_{\mathbf{1}}$, $\mathbf{A}_{\mathbf{2}}$, and $\mathbf{A}_{\mathbf{3}}$ contain structural parameters with dimensions conformable with $\mathbf{y}_{\mathbf{t}}$ and $\mathbf{x}_{\mathbf{t}}$ as needed. An alternative specification would have the expectations in (1) formed using period $\mathrm{t}$ rather than period $\mathrm{t}-1$ information. More will be said about this alternative specification later, particularly in section 4. Specification (1) is more general than may first appear. Models with complicated lag and lead structures can be manipulated into this form (Binder and Pesaran, 1995). Variables that are predetermined and time changes in policy instruments can be included in $\mathbf{y}_{\mathbf{t}}$.

Assumption one: The instrument vector, $\mathbf{x}_{\mathbf{t}}$, is set as a linear function of the state vector, $\mathbf{y}_{\mathrm{t}-1}$.

Policy therefore follows the rule:

$$
\mathbf{x}_{\mathbf{t}}=\Psi_{\mathbf{t}_{-1}}
$$


where the $\mathrm{p} \times \mathrm{n}$ matrix $\Psi$ contains the policy feedback coefficients. Where necessary, lags of the instrument vector enter into this rule through $\mathbf{y}_{\mathbf{t}-1}$. It is desirable to allow some elements of $\Psi$ to equal zero or be otherwise restricted, thereby accommodating simple rules. Let the unrestricted elements of $\Psi$ be represented by the (b×1) vector $\varphi$.

Defining $\mathbf{z}_{\mathbf{t}}=\left[\mathbf{y}_{\mathbf{t}}{ }^{\mathrm{T}} \mathbf{x}_{\mathbf{t}}^{\mathrm{T}}\right]^{\mathrm{T}}$ and combining (1) and (2) produces the system:

$$
\mathbf{B}_{0} \mathbf{z}_{t}=\mathbf{B}_{1} \mathbf{z}_{t-1}+\mathbf{B}_{2} \mathrm{E}_{\mathrm{t}-1} \mathbf{z}_{t+1}+\mathbf{u}_{\mathrm{t}} . \quad \quad \mathbf{u}_{\mathrm{t}} \sim \operatorname{iid}[\mathbf{0}, \Xi]
$$

Clearly $\mathbf{z}_{\mathbf{t}}$ has dimensions $(n+p) \times 1$, and hence $\Omega$ and $\mathbf{W}$ are $(n+p) \times(n+p)$ matrices. The central bank's behavior is formalized as follows:

Assumption two: The monetary authority operates under the regime: $\mathbf{W}, \mathbf{z}_{\mathbf{t}}^{*}=\mathbf{0} \forall \mathrm{t}$, and selects the unique $\varphi \in \mathrm{Q} \subset \mathfrak{R}^{\mathrm{b}}$, that minimizes $\operatorname{Loss}[0, \infty]=\operatorname{tr}[\mathbf{W} \Omega]$, subject to (3).

\section{3) Identifying the Policy Preference Matrix}

This section is central to the paper. It provides necessary and sufficient conditions for identification of the policy preference matrix $\mathbf{W}$. Before turning to the details of these identification conditions, which are presented in a sequence of propositions, it is useful to underline from the outset what is known and what is to be determined.

Substituting (2) into (1) gives:

$$
A_{0} \mathbf{y}_{t}=\left(A_{1}+A_{3} \Psi\right) \mathbf{y}_{t-1}+A_{2} E_{t-1} y_{t+1}+\mathbf{v}_{t}
$$

The solution to (4) takes the form (see McCallum, 1983, or Uhlig, 1999): 
$\mathbf{y}_{\mathrm{t}}=\Pi_{1} \mathbf{y}_{\mathrm{t}-1}+\Pi_{2} \mathbf{v}_{\mathrm{t}}$

where $\Pi_{2}=\mathbf{A}_{0}^{-1}$ and $\Pi_{1}$ satisfies:

$$
\mathbf{A}_{0} \Pi_{1}=A_{1}+A_{3} \Psi+A_{2} \Pi_{1}^{2}
$$

It is assumed that the reduced form parameters, $\Pi_{1}$, and the feedback coefficients in the policy rule, $\Psi$, are known.

Identification problems arise on several levels: first because the system is simultaneous; second because rational expectations terms are present; and third because the system is subject to control. As a consequence the identification strategy proposed below is a recursive one. At its most simplistic the identification problem is one of imposing enough structure on the system so that estimates of the structural parameters can be backed out from the reduced form. ${ }^{5}$

For ease of exposition, define:

$$
\mathbf{C} \stackrel{\text { def }}{=} \mathbf{A}_{1}+\mathbf{A}_{3} \mathbf{\Psi}
$$

This $\mathbf{C}$ matrix is a commingling of the parameters applied to the state vector in equation (4). Now, variance-covariance matrix restrictions aside, (6) implies:

$$
\left[\begin{array}{lll}
\mathbf{A}_{0} & -\mathbf{C} & -\mathbf{A}_{2}
\end{array}\right]\left[\begin{array}{c}
\boldsymbol{\Pi}_{1} \\
\mathbf{I} \\
\mathbf{\Pi}_{1}^{2}
\end{array}\right] \stackrel{\text { def }}{=}[\mathbf{H}][\mathbf{\Gamma}]=[\mathbf{0}],
$$

where, in matrix form

5 Identification of simultaneous systems is analyzed thoroughly in Fisher (1966). Pesaran (1988) examines identification in rational expectations models. 
$[\mathbf{C}]=\left[\begin{array}{ll}\mathbf{A}_{1} & \mathbf{A}_{3}\end{array}\right]\left[\begin{array}{l}\mathbf{I} \\ \mathbf{\Psi}\end{array}\right] \stackrel{\operatorname{def}}{=}[\mathbf{A}][\mathbf{\Lambda}]$

\section{1) Step One}

The first step in the recursive identification strategy involves identifying $\mathbf{H}$ in equation (7). Necessary and sufficient conditions for identifying $\mathbf{H}$ are summarized in:

Proposition one: Let the parameters in the i'th row of $\mathbf{H}, \mathbf{h}_{\mathbf{i}}(1 \times 3 \mathrm{n})$, be subject to ' $\mathrm{r}_{\mathrm{i}}$ ' linear inhomogeneous restrictions, $\mathbf{h}_{\mathbf{i}} \mathbf{R}_{\mathbf{i}}=\mathbf{r}_{\mathbf{i}}$, where $\mathbf{R}_{\mathbf{i}}$ has dimensions $\left(3 n \times r_{i}\right)$, then a necessary condition for identifying $\mathbf{H}$ is $\mathrm{r}_{\mathrm{i}} \geq 2 \mathrm{n}, \forall \mathrm{i} \in[1, \ldots, \mathrm{n}]$. A sufficient condition for identifying $\mathbf{H}$ is $\operatorname{rank}\left[\Gamma \mathbf{R}_{\mathbf{i}}\right]=3 \mathrm{n}, \forall \mathrm{i} \in[1, \ldots, \mathrm{n}]$.

\section{Proof:}

The row vector $\mathbf{h}_{\mathbf{i}}$ is subject to the following linear inhomogeneous restrictions:

$\left[\mathbf{h}_{\mathbf{i}}\right]\left[\mathbf{R}_{\mathbf{i}}\right]=\left[\mathbf{r}_{\mathbf{i}}\right]$.

Combining (7) with (9) produces:

$\left[\mathbf{h}_{\mathrm{i}}\right]\left[\begin{array}{ll}\boldsymbol{\Gamma} & \mathbf{R}_{\mathbf{i}}\end{array}\right]=\left[\begin{array}{ll}\mathbf{0} & \mathbf{r}_{\mathbf{i}}\end{array}\right]$

The dimensions of $\mathbf{h}_{\mathbf{i}}, \Gamma$, and $\mathbf{R}_{\mathbf{i}}$ are $1 \times 3 n, 3 n \times n$, and $3 n \times r_{i}$ respectively. Accordingly, $\mathbf{h}_{\mathbf{i}}$ contains $3 n$ parameters jointly subject to $n+r_{i}$ restrictions. The restrictions in (9) include the normalization restriction arising when a dependent variable is chosen. Consequently, identifying $\mathbf{h}_{\mathbf{i}}$ necessarily requires $r_{i} \geq 2 n$. For these linear inhomogeneous restrictions to be sufficient requires $\left[\Gamma \mathbf{R}_{\mathbf{i}}\right]$ to be such that $\operatorname{rank}\left[\Gamma \mathbf{R}_{\mathbf{i}}\right]=3 \mathrm{n}$ (see Fisher, 1966). 
Proposition one is very intuitive. In a standard simultaneous equations system without rational expectations identifying an equation requires at least as many restrictions be imposed as there are endogenous variables $\left(r_{i} \geq n\right)$. With the rational expectations term present each equation has an additional ' $n$ ' parameters to identify, but the number of reduced form parameters available is unchanged. It directly follows that ' $n$ ' additional restrictions must be imposed to achieve identification.

\section{2) Step Two}

While proposition one provides conditions under which $\mathbf{H}$ is identified, and identification of $\mathbf{H}$ implies identification of $\mathbf{C}$, it does not separately identify $\mathbf{A}_{\mathbf{1}}$ and $\mathbf{A}_{\mathbf{3}}$. This leads to:

Proposition two: Let the $\mathrm{i}$ 'th row of $\mathbf{A}, \mathbf{a}_{\mathbf{i}}(1 \times(\mathrm{n}+\mathrm{p}))$, be subject to ' $\mathrm{q}_{\mathrm{i}}$ ' linear inhomogeneous restrictions, $\mathbf{a}_{i} \mathbf{Q}_{\mathbf{i}}=\mathbf{q}_{i}$, where $\mathbf{Q}_{\mathbf{i}}$ has dimensions $\left((\mathrm{n}+\mathrm{p}) \times \mathrm{q}_{\mathrm{i}}\right)$, then a necessary condition for identifying $\mathbf{A}$ is $\mathrm{q}_{\mathrm{i}} \geq \mathrm{p}, \forall \mathrm{i} \in[1, \ldots, \mathrm{n}]$. A sufficient condition for identifying $\mathbf{A}$ is that $\operatorname{rank}\left[\Lambda \mathbf{Q}_{\mathrm{i}}\right]=\mathrm{n}+\mathrm{p}, \forall \mathrm{i} \in[1, \ldots, \mathrm{n}]$.

Proof:

From equation (8):

$[\mathbf{A}][\mathbf{\Lambda}]=[\mathbf{C}]$.

Assume further that $\mathbf{a}_{\mathbf{i}}$ is subject to ' $\mathrm{q}_{\mathrm{i}}$ ' restrictions of the form:

$\left[\mathbf{a}_{\mathbf{i}}\right]\left[\mathbf{Q}_{\mathbf{i}}\right]=\left[\mathbf{q}_{\mathbf{i}}\right]$.

Combining (10) and (11) gives:

$\left[\mathbf{a}_{\mathrm{i}}\right]\left[\begin{array}{ll}\boldsymbol{\Lambda} & \mathbf{Q}_{\mathrm{i}}\end{array}\right]=\left[\begin{array}{ll}\mathbf{C}_{\mathbf{i}} & \mathbf{q}_{\mathrm{i}}\end{array}\right]$. 
The dimensions of $\mathbf{a}_{\mathbf{i}}, \Lambda$, and $\mathbf{Q}_{\mathbf{i}}$ are $1 \times(n+p),(n+p) \times n$, and $(n+p) \times q_{i}$ respectively. Therefore $\mathbf{a}_{\mathbf{i}}$ contains $\mathrm{n}+\mathrm{p}$ parameters that are collectively subject to $n+q_{i}$ linear restrictions. Consequently, a necessary condition for identification is $q_{i} \geq p$. A sufficient condition for identification of $\mathbf{a}_{\mathbf{i}}$ is $\operatorname{rank}\left[\Lambda \mathbf{Q}_{\mathbf{i}}\right]=\mathrm{n}+\mathrm{p}$.

In terms of the recursive identification strategy the role of proposition two is to disentangle the elements in $\mathbf{A}_{3}$ from those in $\mathbf{A}_{\mathbf{1}}$. The elements in these two matrices are mingled because monetary policy is set conditional upon the state vector. In words propositions two states that a necessary condition for identification is that a restriction on the elements in $\mathbf{A}_{\mathbf{1}}$ and $\mathbf{A}_{\mathbf{3}}$ be imposed for each control variable in the system. Clearly if an equation contains all state variables and an instrument is set as a linear function of all of the state variables, then the coefficient in $\mathbf{A}_{\mathbf{3}}$ associated with that instrument in that equation cannot be identified.

\section{3) Step Three}

Thus far in the identification strategy information contained in the policy feedback matrix $\Psi$ has not been used. As long as the rank conditions of proposition one and two hold, then information in the reduced form coefficients, $\Pi_{1}$ is sufficient to identify all the coefficients in the structural model. In this final identification step we introduce $\Psi$. If $\Psi$ is determined optimally, then its elements will be nonlinear functions of the structural parameters $\left(\mathbf{A}_{\mathbf{0}}, \mathbf{A}_{\mathbf{1}}, \mathbf{A}_{\mathbf{2}}\right.$, and $\mathbf{A}_{\mathbf{3}}$ ), and also $\mathbf{W}$. Provided the structural parameters are identified a crucial ingredient in $\Psi$ is known. This third and final step establishes necessary and sufficient conditions under which knowledge of $\Psi$ and the structural parameters can be used to identify $\mathbf{W}$. These conditions are summarized in:

Proposition three: Let the column vector $\mathbf{w}=\operatorname{vech}(\mathbf{W})$ be subject to 's' linear inhomogeneous restrictions, $\mathbf{S}^{\mathrm{T}} \mathbf{w}=\mathbf{s}$, then a necessary condition for global 
identification of $\mathbf{W}$ is: $s \geq \frac{(n+p)(n+p+1)}{2}-b$. A sufficient condition for global identification of $\mathbf{W}$ is: $\operatorname{rank}\left[\mathbf{J}\left(\mathbf{w}^{* *}\right)^{\mathrm{T}} \mathbf{S}\right]^{\mathrm{T}}=\mathrm{k}=[(\mathrm{n}+\mathrm{p})(\mathrm{n}+\mathrm{p}+1) / 2], \forall \mathbf{w}^{* *} \in \mathrm{P}$ $\subset \mathfrak{R}^{\mathrm{k}}{ }_{+}, \mathrm{P}$ convex, where $\mathbf{J}\left(\mathbf{w}^{* *}\right)$ is the Jacobian of the transform $\mathrm{f}: \mathrm{P} \rightarrow \mathrm{Q}$ defined below.

\section{Proof:}

In its most general form $\mathbf{W}$ is a square, symmetric, matrix containing $(n+p)^{2}$ parameters. Symmetry reduces the number of independent parameters in $\mathbf{W}$ to $[(n+p)(n+p+1)] / 2$. In what follows let $k=[(n+p)(n+p+1) / 2]$.

An outcome of the policy optimization is a continuously differentiable function $\mathrm{f}: \mathrm{P} \rightarrow \mathrm{Q}$ relating the policy preferences to the coefficients in the policy rule: ${ }^{6}$

$\varphi=\mathrm{f}(\mathbf{w})$

Recall that $\varphi$ is a $(b \times 1)$ vector containing the elements of $\Psi$ that are unrestricted. $\mathbf{w}$ is also subject to 's' linear inhomogeneous restrictions of the form:

$\mathbf{S}^{\mathrm{T}} \mathbf{w}=\mathbf{S}$

The policy objective function is only defined up to a scalar allowing one element of $\mathbf{w}$ to be normalized upon. This normalizing restriction is subsumed into (13). Remaining restrictions on $\mathbf{w}$ are most likely to take the form of exclusion restrictions, particularly on the covariance elements of $\Omega$. The non-linearity of (12) complicates identification. From the mean value theorem there exists a $\mathbf{w}^{* *}$ between $\mathbf{w}^{*}$ and $\mathbf{w}$, each elements of $\mathrm{P}$, such that:

\footnotetext{
${ }^{6}$ The structural parameters have been subsumed into the functional form.
} 
$\varphi^{*}=\varphi+\mathbf{J}\left(\mathbf{w}^{* * *}\right)\left(\mathbf{w}^{*}-\mathbf{w}\right)$,

where $\mathbf{J}\left(\mathbf{w}^{* *}\right)$ is an $\mathrm{b} \times \mathrm{k}$ Jacobian matrix. Combining (13) and (14) allows the restrictions on $\mathbf{w}$ to be represented as:

$\left[\begin{array}{c}\mathbf{J}\left(\mathbf{w}^{* *}\right) \\ \mathbf{S}^{\mathrm{T}}\end{array}\right] \mathbf{w}=\left[\begin{array}{c}\boldsymbol{\varphi}-\boldsymbol{\varphi}^{*}+\mathbf{J}\left(\mathbf{w}^{* * *}\right) \mathbf{w}^{*} \\ \mathbf{S}\end{array}\right]$

From (15) a sufficient condition for global identification of $\mathbf{w}$ is $\operatorname{rank}\left[\mathbf{J}\left(\mathbf{w}^{* *}\right)^{\mathrm{T}} \mathbf{S}\right]^{\mathrm{T}}=\mathrm{k}, \forall$ $\mathbf{w}^{* *} \in \mathrm{P}$ (see Rothenberg, 1971). Notice, however, that $\forall \mathrm{n}, \mathrm{p}>0, \mathrm{k}>\mathrm{np} \geq \mathrm{b}$. Therefore, $\operatorname{rank}\left[\mathbf{J}\left(\mathbf{w}^{* *}\right)\right]$ is at most ' $b$ ', which implies that a necessary condition for global identification is $\mathrm{s} \geq \mathrm{k}-\mathrm{b}$, or after substituting for $\mathrm{k}, \mathrm{s} \geq \frac{(\mathrm{n}+\mathrm{p})(\mathrm{n}+\mathrm{p}+1)}{2}-\mathrm{b}$.

The necessary condition of proposition three has a clear interpretation: the ' $b$ ' coefficients in $\varphi$ can be used to identify at most ' $b$ ' elements in $\mathbf{W}$. An interesting aspect of proposition three is that the Jacobian matrix $\mathbf{J}\left(\mathbf{w}^{* *}\right)$ itself need not have full rank for all $\mathbf{w}^{* *} \in \mathrm{P}$. A singularity in the Jacobian matrix means that there is no information in the functional relationship between $\mathbf{w}$ and $\varphi$ to tie down one or more parameters in $\mathbf{w}$. However, provided this lack of information in $\mathbf{J}\left(\mathbf{w}^{* *}\right)$ can be offset by additional outside information in the form of additional columns in $\mathbf{S}$ identification is still possible.

\section{4) In Addition...}

Of course equation (2) implies that the relationship between the policy instruments and the predetermined variables is a deterministic one. Rarely would this be the case. In practice the information set used by agents to form their expectations, and that an econometrician uses when estimating policy reaction functions, may only be a subset of that available to the monetary authority when it sets policy. This can arise if the monetary authority uses a more recent information set than other agents. Where this is 
the case deviations between the actual path of $\mathbf{x}_{\mathbf{t}}$ and that predicted by (2) are to be expected. These deviations are accommodated by adding a $\mathrm{p} \times 1$ innovation vector $\mathbf{e}_{\mathbf{t}}$, uncorrelated with $\mathbf{v}_{\mathbf{t}}$ and $\mathbf{y}_{\mathbf{t}-\mathbf{1}}$, to equation (2) giving:

$\mathbf{x}_{t}=\Psi_{\mathbf{t}_{-1}}+\mathbf{e}_{\mathrm{t}}$

Intuitively, adding this innovation vector facilitates identification of $\mathbf{A}_{\mathbf{1}}$ and $\mathbf{A}_{\mathbf{3}}$ because it automatically imbues $\mathbf{x}_{\mathbf{t}}$ with volatility absent from $\mathbf{y}_{\mathbf{t}-1}$. The addition of the policy innovation term leads to:

Proposition four: Given (1) and (16) $\mathbf{A}_{\mathbf{1}}$ and $\mathbf{A}_{\mathbf{3}}$ are identified provided the rank condition of proposition one holds.

\section{Proof:}

Substituting (16) into (1) produces:

$$
\mathbf{A}_{0} \mathbf{y}_{t}=\left(\mathbf{A}_{1}+\mathbf{A}_{3} \Psi\right) \mathbf{y}_{t-1}+\mathbf{A}_{2} \mathrm{E}_{\mathrm{t}-1} \mathbf{y}_{t+1}+\mathbf{A}_{3} \mathbf{e}_{\mathrm{t}}+\mathbf{v}_{\mathbf{t}}
$$

Equation (16) is identified because it contains only predetermined variables and therefore both $\Psi$ and $\mathbf{e}_{\mathbf{t}}$ are assumed to be known. The rational expectations solution to (17) takes the form:

$\mathbf{y}_{t}=\Pi_{1} \mathbf{y}_{t-1}+\Pi_{2} v_{t}+\Pi_{3} e_{t}$

where $\Pi_{2}=\mathbf{A}_{0}{ }^{-1}, \Pi_{1}$ satisfies (6), and $\Pi_{3}=\mathbf{A}_{0}{ }^{-1} \mathbf{A}_{3}$. The reduced form parameter matrices $\Pi_{1}$ and $\Pi_{3}$ are known and the solution to (17) asserts that once $\mathbf{A}_{\mathbf{0}}$ is identified so too is A3. Proposition one presents a sufficient condition for $\mathbf{A}_{\mathbf{0}}$ to be identified. 
Finally, we may wonder how these identification conditions would be affected if the expectations in (1) were formed using period t rather than period t-1 information. It is not difficult to show that provided variance-covariance matrix restrictions are not used for identification, and provided the policy rule continues to depend only on $\mathbf{y}_{\mathbf{t}-\mathbf{1}}$ and does not contain $\mathbf{v}_{\mathbf{t}}$, propositions one - four remain unaffected. The intuition behind this result is that the solution for $\Pi_{1}$ is unaltered by the change to period $t$ information. The solution for $\Pi_{2}$ does change, but it is not required for identification provided $\mathbf{v}_{\mathbf{t}}$ does not enter the policy rule.

\section{4) Some Examples}

This section takes some popular models from the literature and examines whether they satisfy the conditions necessary for identification of policy regimes. The aims of this section are twofold. First the section aims to illustrate how propositions one, two, and three are applied in practice. Second, the section aims to investigate the suitability of various models as vehicles for identifying policy regimes.

All models considered contain the variables: $y_{t}, \pi_{t}$, and $i_{t}$, representing the output gap, inflation and the nominal interest rate respectively, and as a consequence the policy objective function used throughout this section is taken to be:

$\operatorname{Loss}[0, \infty]=\alpha \operatorname{Var}\left[\pi_{\mathrm{t}}\right]+(1-\alpha) \operatorname{Var}\left[\mathrm{y}_{\mathrm{t}}\right]+\sigma \operatorname{Var}\left[\mathrm{i}_{\mathrm{t}}\right]$

Accordingly, the systems examined require that we identify just two policy preference parameters. As such, for each system $\mathbf{S}$ has four independent columns implying s $=4$.

\section{1) Example One}

Consider the following system: 
$\mathrm{y}_{\mathrm{t}}=\beta \mathrm{y}_{\mathrm{t}-1}-\gamma\left[\mathrm{i}_{\mathrm{t}}-\mathrm{E}_{\mathrm{t}-1} \pi_{\mathrm{t}+1}\right]+\mathrm{g}_{\mathrm{t}}$,

$\pi_{\mathrm{t}}=\pi_{\mathrm{t}-1}+\lambda \mathrm{y}_{\mathrm{t}-1}+\mathrm{u}_{\mathrm{t}}$

$\mathrm{i}_{\mathrm{t}}=\varphi_{\mathrm{y}} \mathrm{y}_{\mathrm{t}-1}+\varphi_{\pi} \pi_{\mathrm{t}-1}$.

Equation (19) is a dynamic IS curve, (20) an accelerationist Phillips curve, and (21) the policy reaction function. The stochastic terms $-g_{t}$ and $u_{t}$ - are assumed to be finite variance white noise processes. Observe first that equations (20) and (21) are identified in so much as for them the rank conditions of propositions one and two hold. For subsequent identification of other coefficients the coefficients in equations (20) and (21) are assumed known. Next note that with two feedback parameters in (21) and two independent policy preference coefficients the necessary condition of proposition three is satisfied. It just remains to be seen whether propositions one and two hold when applied to (19).

In terms of equation (4) an unconstrained representation of (19) takes the form:

$$
\eta_{1} y_{t}=\eta_{2} \pi_{t}+c_{1} y_{t-1}+c_{2} \pi_{t-1}+\rho_{1} E_{t-1} y_{t+1}+\rho_{2} E_{t-1} \pi_{t+1}+g_{t} .
$$

The system (19) - (21) has $n=2$ and $p=1$. Therefore, the order condition of proposition one requires that (19) place at least four restrictions $\left(r_{i} \geq 2 n\right)$ on the structure of (22). Relative to (22), equation (19) imposes: $\eta_{1}=1 ; \eta_{2}=0 ; \rho_{1}=0$; and $\rho_{2}=c_{2} / \varphi_{\pi}$. Thus proposition one's order condition for identification is satisfied.

Proposition two requires the number of restrictions on the elements of $\mathbf{A}_{\mathbf{1}}$ and $\mathbf{A}_{\mathbf{3}}$ associated with the IS curve be greater than or equal to the number of policy instruments. It is useful to rewrite (22) as:

$$
\eta_{1} y_{t}=\eta_{2} \pi_{t}+\lambda_{1} y_{t-1}+\lambda_{2} \pi_{t-1}+\rho_{1} E_{t-1} y_{t+1}+\rho_{2} E_{t-1} \pi_{t+1}+\gamma i_{t}+g_{t} .
$$

In light of (23), the restrictions on the IS curve's structural parameters take the form: 
$\left[\begin{array}{lll}\lambda_{1} & \lambda_{2} & -\gamma\end{array}\right]\left[\begin{array}{ccc}1 & 0 & 0 \\ 0 & 1 & 1 \\ \varphi_{\mathrm{y}} & \varphi_{\pi} & 0\end{array}\right]=\left[\begin{array}{lll}\mathrm{c}_{1} & \mathrm{c}_{2} & 0\end{array}\right]$

Provided $\pi_{\mathrm{t}-1}$ enters the policy reaction function non-trivially $\left(\varphi_{\pi} \neq 0\right)$ the rank condition of proposition two is satisfied. ${ }^{7}$ In this system the policy regime can be identified.

\section{2) Example Two}

The second example is adapted from McCallum (1997) and consists of the following equations for the output gap and inflation:

$$
\begin{aligned}
& \mathrm{y}_{\mathrm{t}}=\mathrm{E}_{\mathrm{t}-1} \mathrm{y}_{\mathrm{t}+1}-\gamma\left[\mathrm{i}_{\mathrm{t}}-\mathrm{E}_{\mathrm{t}-1} \pi_{\mathrm{t}+1}\right]+\mathrm{g}_{\mathrm{t}} \\
& \pi_{\mathrm{t}}=\mathrm{E}_{\mathrm{t}-1} \pi_{\mathrm{t}+1}+\lambda \mathrm{y}_{\mathrm{t}}+\mathrm{u}_{\mathrm{t}} .
\end{aligned}
$$

To add some persistence both $\mathrm{g}_{\mathrm{t}}$ and $\mathrm{u}_{\mathrm{t}}$ are assumed to follow $\mathrm{AR}(1)$ processes:

$$
\left[\begin{array}{l}
g_{t} \\
u_{t}
\end{array}\right]=\left[\begin{array}{cc}
\mu_{1} & 0 \\
0 & \mu_{2}
\end{array}\right]\left[\begin{array}{l}
g_{t-1} \\
u_{t-1}
\end{array}\right]+\left[\begin{array}{c}
- \\
g_{t} \\
- \\
u_{t}
\end{array}\right] .
$$

McCallum's system is completed by the addition of the policy reaction function:

$$
\mathrm{i}_{\mathrm{t}}=\varphi_{\mathrm{g}} \mathrm{g}_{\mathrm{t}-1}+\varphi_{\mathrm{u}} \mathrm{u}_{\mathrm{t}-1}
$$

7 Notice that $\varphi_{\mathrm{y}}$ can equal zero, but not $\varphi_{\pi}$, and the system is still identified. Thus the optimal simple inflation rule $i_{t}=\varphi_{\pi} \pi_{t-1}$ can still be examined. A policy rule where the interest rate responds only to the output gap might usually be expected to lead to nominal indeterminacy. In this model it produces an unidentified system. Moreover, with only one parameter in the policy rule the order condition for proposition three is not met so the two policy preference parameters cannot be identified. 
Analogous to example one the policy reaction function (26) is identified and contains two feedback coefficients. With only two independent parameters in the policy objective function proposition three's order condition is meet. Unfortunately neither (24) nor (25) are identified. In unrestricted form equation (25) has the form:

$$
\begin{aligned}
\eta_{1} \pi_{\mathrm{t}}= & \eta_{2} \mathrm{y}_{\mathrm{t}}+\eta_{3} \mathrm{~g}_{\mathrm{t}}+\eta_{4} \mathrm{u}_{\mathrm{t}}+\lambda_{1} \pi_{\mathrm{t}-1}+\lambda_{2} \mathrm{y}_{\mathrm{t}-1}+\lambda_{3} \mathrm{~g}_{\mathrm{t}-1}+\lambda_{4} \mathrm{u}_{\mathrm{t}-1} \\
& +\rho_{1} \mathrm{E}_{\mathrm{t}-1} \pi_{\mathrm{t}+1}+\rho_{2} \mathrm{E}_{\mathrm{t}-1} \mathrm{y}_{\mathrm{t}+1}+\rho_{3} \mathrm{E}_{\mathrm{t}-1} \mathrm{~g}_{\mathrm{t}+1}+\rho_{4} \mathrm{E}_{\mathrm{t}-1} \mathrm{u}_{\mathrm{t}+1}+\bar{u}_{\mathrm{t}}
\end{aligned}
$$

Comparing equation (25) with (27) the independent restrictions imposed on (27) are: $\eta_{1}=1 ; \eta_{3}=0 ; \eta_{4}=0 ; \lambda_{1}=0 ; \lambda_{2}=0 ; \lambda_{3}=0 ; \rho_{1}=1 ; \rho_{2}=0 ; \rho_{3}=0 ;$ and $\rho_{4}=0$. These restrictions number ten while the order condition of proposition one requires only eight restrictions. In terms of this necessary condition equation (25) is over-identified. Appearances are deceiving, however. For while equation (25) satisfies proposition one's order condition it fails the rank condition. To see this, observe that the restrictions listed exclude $\pi_{\mathrm{t}-1}, \mathrm{y}_{\mathrm{t}-1}$, and $\mathrm{g}_{\mathrm{t}-1}$ from the system. At the same time the rational expectations solution to the system expresses $\pi_{\mathrm{t}}$ and $\mathrm{y}_{\mathrm{t}}$ in terms of just $\mathrm{u}_{\mathrm{t}-1}$, also excluding ${ }^{8} \pi_{\mathrm{t}-1} \mathrm{y}_{\mathrm{t}-1}$, and $\mathrm{g}_{\mathrm{t}-1}$. Thus three of the columns in the $\left[\Gamma \mathbf{R}_{\mathbf{i}}\right]$ matrix associated with (25) depend linearly on the others and $\left[\Gamma \mathbf{R}_{\mathbf{i}}\right]$ has rank $=11<12(3 \mathrm{n}$, where $\mathrm{n}=4)$. In a similar vein it can be shown that equation (24) is also unidentified.

\section{3) Example Three}

Our final example comes from Clarida, Gali and Gertler (1999) and is of the same genre to those just analyzed. It differs, however, in that the persistence in the system is endogenous, determined by lagged dependent variables, and not exogenous, driven by autocorrelated shocks. In fact the shocks $g_{t}$ and $u_{t}$ are assumed to be finite variance white noise processes.

8 That all elements in $\Pi_{1}$ associated with $\pi_{\mathrm{t}-1}$ and $\mathrm{y}_{\mathrm{t}-1}$ equal zero is clear because these two variables do not appear in the system's structure and hence only enter the system's 


$$
\begin{aligned}
& \mathrm{y}_{\mathrm{t}}=\beta \mathrm{y}_{\mathrm{t}-1}+(1-\beta) \mathrm{E}_{\mathrm{t}-1} \mathrm{y}_{\mathrm{t}+1}-\gamma\left[\mathrm{i}_{\mathrm{t}}-\mathrm{E}_{\mathrm{t}-1} \pi_{\mathrm{t}+1}\right]+\mathrm{g}_{\mathrm{t}} \\
& \pi_{\mathrm{t}}=\delta \pi_{\mathrm{t}-1}+(1-\delta) \mathrm{E}_{\mathrm{t}-1} \pi_{\mathrm{t}+1}+\lambda \mathrm{y}_{\mathrm{t}}+\mathrm{u}_{\mathrm{t}} \\
& \mathrm{i}_{\mathrm{t}}=\varphi_{\mathrm{y}} \mathrm{y}_{\mathrm{t}-1}+\varphi_{\pi} \pi_{\mathrm{t}-1}
\end{aligned}
$$

Like the previous two examples the order condition of proposition three is satisfied because the policy reaction function contains two feedback coefficients. Now consider equation (28). In the structure of equation (4), at its most general, equation (28) becomes:

$$
\eta_{1} y_{t}=\eta_{2} \pi_{t}+c_{1} y_{t-1}+c_{2} \pi_{t-1}+\rho_{1} E_{t-1} y_{t+1}+\rho_{2} E_{t-1} \pi_{t+1}+g_{t}
$$

Relative to (28) equation (30) imposes the four restrictions: $\eta_{1}=1 ; \eta_{2}=0 ; c_{2}=-\rho_{2} \varphi_{\pi}$; and $c_{1}=1-\rho_{1}-\rho_{2} \varphi_{y}$, which with $n=2$ means that the order condition of proposition one is met. Provided neither $\beta$ nor $\delta$ equal zero the rank condition is also satisfied. ${ }^{9}$ Moreover, if $\varphi_{\pi}$ is non-zero the rank condition of proposition two is also satisfied implying that equation (28) is identified. If $\varphi_{\pi}$ does equal zero, then the order condition of proposition three does not hold and the policy preference parameters cannot be identified.

Now consider the Phillips curve, equation (29). The unrestricted Phillips curve is:

$$
\eta_{2} \pi_{t}=\eta_{1} y_{t}+c_{1} y_{t-1}+c_{2} \pi_{t-1}+\rho_{1} E_{t-1} y_{t+1}+\rho_{2} E_{t-1} \pi_{t+1}+u_{t}
$$

Relative to (31) equation (29) imposes the restrictions: $\eta_{2}=1 ; c_{1}=0 ; c_{2}+\rho_{2}=1$; and $\rho_{1}$ $=0$. These four restrictions satisfy proposition one's necessary condition for identification. Like the IS curve (28), provided neither $\beta$ nor $\delta$ equal zero the rank

state vector trivially. That the elements associated with $\mathrm{g}_{\mathrm{t}-1}$ also equal zero (or for one equation $\mu_{1}$ ) is a consequence of the system being subject to control.

9 When $\beta=0$, for example, $\mathrm{y}_{\mathrm{t}-1}$ is not a state variable in the system and $\varphi_{\mathrm{y}}$ appropriately equals zero. Consequently, the column of $\Pi_{1}$ associated with $\mathrm{y}_{\mathrm{t}-1}$ equals zero, leading to the rank condition of proposition one failing. 
condition of proposition one is also met. As a consequence for this system the policy regime is identifiable.

What these three examples illustrate is that provided we consider only the order condition for identifying the policy preferences (proposition three) the major obstacle faced when identifying $\mathbf{W}$ is that of identifying the structure of the economy. Identifying the economy's structure is essential, however, because it constrains the optimization process leading to the policy rule.

\section{5) Conclusions}

The aim of this paper was simple. We wanted to lay the foundations for estimating central bank policy preferences by establishing conditions under which these preference parameters could be identified. It was demonstrated that optimizing central banks apply policy rules whose feedback coefficients are nonlinear functions of its policy preferences. Before these policy preferences can be backed out from these feedback coefficients several identification conditions need to hold. As a consequence this paper proposes a recursive identification strategy consisting of three steps. The first two steps, summarized in propositions one and two, identify the parameters in the structural model constraining the central bank's optimization. Only once the structural model is identified can enough structure be placed on the policy reaction function to disentangle the policy preference coefficients. Proposition three provides necessary and sufficient conditions for the policy preference coefficients to be identified.

To illustrate how the conditions developed in propositions one, two and three are applied in practice three examples were provided and their identification properties examined. The identification conditions developed in this paper are important because only by identifying and estimating the policy regime in operation can we tell what the objectives of the monetary authority truly are. In particular, this paper serves to emphasize that it is not necessarily possible to say anything meaningful about a policy regime purely on the 
basis of an estimated policy rule. Future work will seek to identify and estimate actual policy regimes in operation, and to document how these policy regimes have changed over time. 


\section{References}

Ball, L., (1999), 'Efficient Rules for Monetary Policy', International Finance, 2, 1, pp6383.

Bharucha, N., and C. Kent, (1998), 'Inflation Targeting in a Small Open Economy' Reserve Bank of Australia, RDP \#9807.

Binder, M., and H. Pesaran, (1995), 'Multivariate Rational Expectations Models and Macroeconomic Modeling: A Review and Some New Results', in Pesaran, H. and M. Wickens (eds), Handbook of Applied Econometrics.

Clarida, R., J. Gali, and M. Gertler, (1998), 'Monetary Policy Rules in Practice: Some International Evidence', European Economic Review, 42, pp1033-1067.

Clarida, R., J. Gali, and M. Gertler, (1999), 'The Science of Monetary Policy: A New Keynesian Perspective', Journal of Economic Literature, forthcoming.

Fair, R., and P. Howrey, (1996), 'Evaluating Alternative Monetary Policy Rules' Journal of Monetary Economics, 38, pp173-193.

Fisher, F., (1966), The Identification Problem in Econometrics, McGraw-Hill Inc, New York.

Fuhrer, J., (1997), 'Inflation/Output Variance Trade-Offs and Optimal Monetary Policy', Journal of Money, Credit, and Banking, 29, 2, pp214-234.

Judd, J., and G. Rudebusch, (1998), 'Taylor's Rule and the Fed: 1970-1997', Federal Reserve Bank of San Francisco, Economic Review, 3, pp3-16.

McCallum, B., (1983), 'On Non-Uniqueness in Rational Expectations Models: An Attempt at Perspective', Journal of Monetary Economics, 11, pp139-168.

Pesaran, H., (1988), 'Identification of Rational Expectations Models', Journal of Econometrics, 16, pp375-398.

Rothenberg, T., (1971), 'Identification in Parametric Models', Econometrica, 39, 3, pp577-591.

Rudebusch, G., and L. Svensson, (1998), 'Policy Rules for Inflation Targeting' NBER working paper, \#6512, forthcoming in Taylor (ed) Monetary Policy Rules, Chicago University Press.

Soderlind, P., (1999), 'Solution and Estimation of RE Macromodels with Optimal Policy', European Economic Review, 43, pp813-823. 
Svensson, L., (1998), 'Open-Economy Inflation Targeting', Journal of International Economics, Forthcoming.

Svensson, L., (1999), 'Inflation Targeting as a Monetary Policy Rule', Journal of Monetary Economics, 43, 3, pp607-654.

Taylor, J., (1993), 'Discretion versus Policy Rules in Practice', Carnegie-Rochester Conference Series on Public Policy, 39, pp195-214.

Uhlig, H., (1999), 'A Toolkit for Analysing Nonlinear Dynamic Stochastic Models Easily', in Marimon, R., and A. Scott (eds), Computational Methods for the Study of Dynamic Economies, Oxford University Press, New York. 